\title{
Supporting Information for "Anomalous
}

\section{Temperature Dependence of Exciton}

\section{Spectral Diffusion in Tetracene Thin Film"}

\author{
Tatsuya Yoshida, ${ }^{\dagger}$ Kazuya Watanabe, ${ }^{*} \dagger$ Marin Petrović, ${ }^{\ddagger}$ and Marko Kralj‡ \\ Department of Chemistry, Graduate School of Science, Kyoto University, Kyoto, \\ 606-8502, Japan, and Center of Excellence for Advanced Materials and Sensing \\ Devices, Institute of Physics, Bijenička 46, 10000 Zagreb, Croatia
}

E-mail: kw@kuchem.kyoto-u.ac.jp

*To whom correspondence should be addressed

${ }^{\dagger}$ Department of Chemistry, Graduate School of Science, Kyoto University, Kyoto, 606-8502, Japan

${ }^{\ddagger}$ Center of Excellence for Advanced Materials and Sensing Devices, Institute of Physics, Bijenička 46, 10000 Zagreb, Croatia 


\section{Contents}

1 Experimental setup $\quad$ S3

2 2DES measurement $\quad$ S5

3 Characterizations of the tetracene film by a low-energy electron diffraction, ultraviolet photoemission, and photoluminescence spectroscopy S9

4 Numerical simulations of the optical reflectivity of a tetracene thin filmS12

5 Temperature dependence of LEED pattern $\quad$ S14

6 Estimation of $N_{c o h}$ by a two-dimensional Frenkel exciton model S16

7 Numerical simulation of the 2DES spectra used for deducing FFCF $\begin{array}{ll}\text { parameters } & \text { S17 }\end{array}$

8 Anharmonic coupling model of the correlation function $\quad$ S20 


\section{Experimental setup}

Figure S1 shows a schematic illustration of the experimental setup. All the measurements were carried out in ultrahigh vacuum (UHV) with base pressure lower than $3 \times 10^{-8}$ Pa. ${ }^{1,2}$ The tetracene thin film was deposited on a graphene covered $\operatorname{Ir}(111)$ prepared by the following procedure. A single crystal $\operatorname{Ir}(111)$ surface $(10 \mathrm{~mm}$ in diameter, Metek) was cleaned by cycles of $\mathrm{Ar}^{+}$sputtering and subsequent annealing to $1500 \mathrm{~K}$ followed by $\mathrm{O}_{2}$ treatment at $1200 \mathrm{~K}$. A graphene monolayer was fabricated on the $\operatorname{Ir}(111)$ by chemical vapor deposition of acetylene. ${ }^{3}$ The quality of the graphene was confirmed by an ultraviolet photoemission spectroscopy and a low energy electron diffraction (see SI, section 3 for the details). The sample temperature was controlled between $95 \mathrm{~K}$ and $1500 \mathrm{~K}$ by cooling with liquid $\mathrm{N}_{2}$ and heating with an electron bombardment, respectively. The temperature was measured by a thermocouple attached to a molybdenum holder close to the Ir substrate. The thermal deposition of tetracene (Nakarai tesque, $98 \%$ ) was carried out with a quartz effusive cell ('evaporator' in Figure S1) resistively heated with a tungsten wire. The temperature of the graphene on $\operatorname{Ir}(111)$ was kept at $\sim 298 \mathrm{~K}$ during the deposition. The optical reflectivity of the $p$-polarized light derived from a halogen lamp was monitored as a function of the tetracene deposition time by using a charge-coupled device camera (Andor DV420A-BU3) attached to a spectrometer (SOLAR T2 MS3504i). The incident angle of the light was $\sim 67^{\circ}$. The average deposition rate was estimated to be $0.4 \mathrm{~nm} / \mathrm{min}$. 


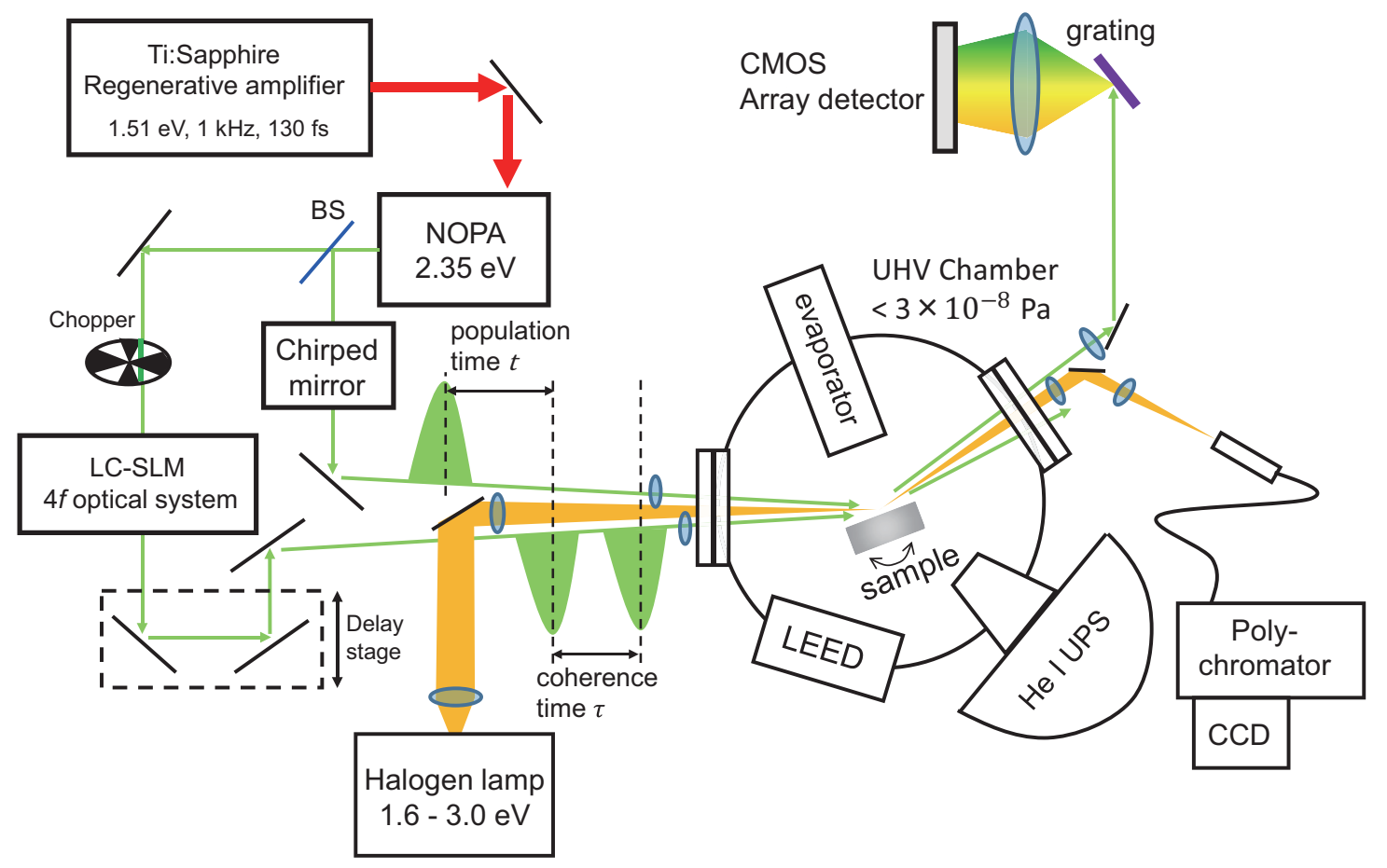

Figure S1: A schematic illustration of the experimental setup. 


\section{2DES measurement}

The 2DES measurement was carried out by using a home-build non-collinear optical parametric amplifier (NOPA) $)^{1,2,4,5}$ pumped by a second harmonics output of a Ti:sapphire regenerative amplifier (Spectra physics Spitfire-Pro, $1 \mathrm{kHz}$ ). The output of the NOPA was tuned at $2.35 \mathrm{eV}$ to resonantly excite the lowest absorption peak of the tetracene film. While the major part of the NOPA output was converted into a phase-locked pulse-pair, a part of the pulse was used as a time-delayed probe pulse. 2D-ES was carried out by measuring the probe reflectance change for various relative phase and delay (coherence time $\tau$ ) between the phase-locked pump pair as a function of the population time $t$, the time delay between the pump-pair and the probe.

The phase-locked pulse pair was synthesized by a standard pulse shaping technique following the literature. ${ }^{6}$ We used a folded $4-f$ optical system ${ }^{7}$ in which a liquid crystal spatial light modulator (JENOPTIK SLM-S320d) is placed at the Fourier plane (Figure $\mathrm{S} 2$ ). In the frequency domain, the shaping system imposes a mask function $M(\omega)$ on the input pulse $E_{\text {in }}(\omega)$ that results in an output field $E_{\text {out }}(\omega)$ as, ${ }^{6,8}$

$$
E_{\text {out }}(\omega)=M(\omega) E_{\text {in }}(\omega)
$$

For converting a laser pulse into a pulse-pair, $M(\omega)$ is given by

$$
M(\omega)=\frac{1}{2} \cdot\left[\exp (i \omega \tau) \cdot \exp \left(i \phi_{1}\right)+\exp \left(i \phi_{2}\right)\right]
$$

where $\tau$ is a time-interval of the pulse pair that corresponds to the coherence time in 2DES measurement; $\phi_{1}\left(\phi_{2}\right)$ stands for a phase of the first (second) pulse.

The details of the technique for varying the phase and amplitude of the input field have been well documented in literature. ${ }^{6}$ Figure S2 shows a schematic illustration of the liquid-crystal spatial light modulator (SLM). The SLM consists of two liquid crystal display with 320 stripe electrodes on each display. On the Fourier plane of the $4-f$ setup, each frequency component of the light is focused on one of the electrodes. The optical 


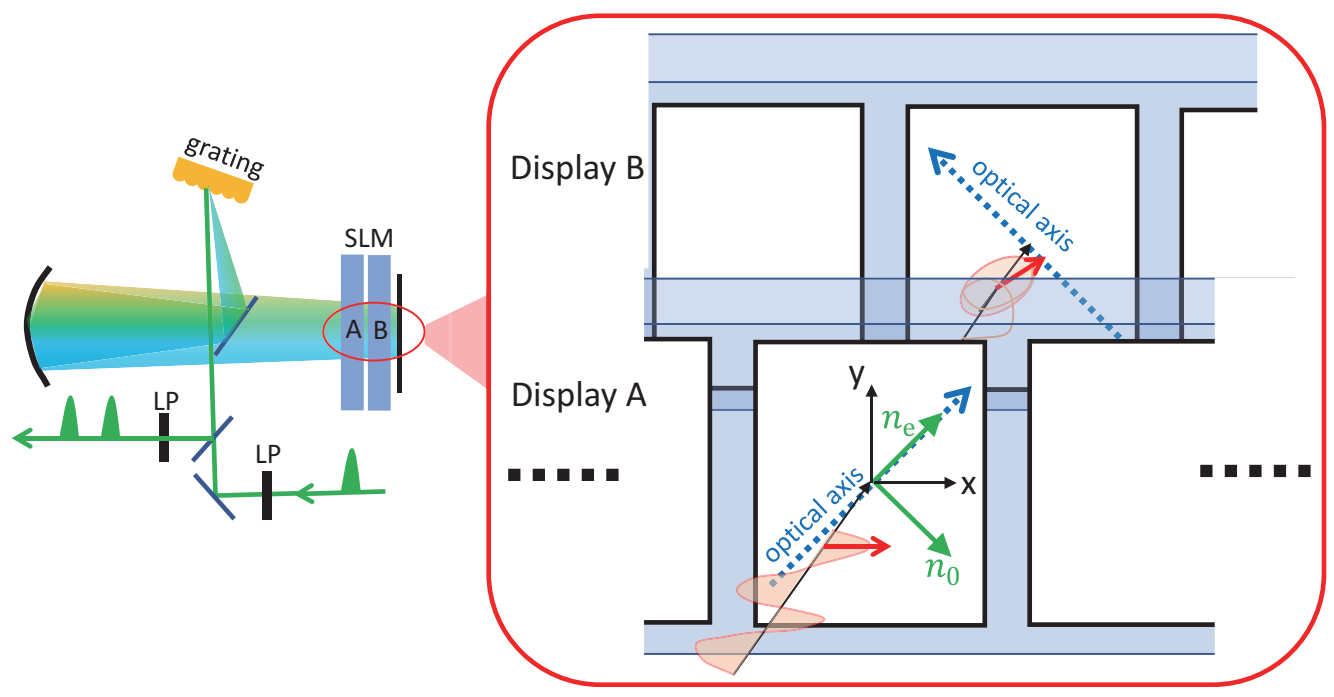

Figure S2: Schematic illustration of the pulse shaping part of the optical setup. (Left) a folded 4 -foptical setup in which a single pulse is converted into a phase-locked pulse pair. LP: linear polarizer. (Right) a schematic illustration of the two liquid crystal display of the SLM. The white boxes indicate striped electrodes. $x$-polarized electromagnetic wave is converted to an elliptically polarized wave via the birefringence in the liquid crystal.

axis of each liquid crystal is aligned to be orthogonal to each other between the display $\mathrm{A}$ and $\mathrm{B}$. The phase difference $\Delta \theta$ between the ordinary and the extraordinary lights passing through the liquid crystal is given by,

$$
\Delta \theta=\frac{2 \pi\left(n_{\mathrm{e}}-n_{\mathrm{o}}\right) d}{\lambda}
$$

where $n_{\mathrm{o}}\left(n_{\mathrm{e}}\right)$ is an (extra)ordinary refractive index of the liquid crystal; $d$ is the thickness of the crystal; $\lambda$ is the wavelength of the light.

Since $n_{\mathrm{e}}$ of each pixel can be controlled by varying the bias voltage, $\Delta \theta$ for each pixel in display $\mathrm{A}$ and $\mathrm{B}$ can be varied independently. If we consider an incidence of linearly polarized light with $x$-component of the electric field, the intensity and phase of the light transmitted through the pair of the liquid crystal plates and a linear polarizer (see Figure S2) vary as a function of $\Delta \theta$ of display $\mathrm{A}$ and $\mathrm{B}\left(\Delta \theta_{A}\right.$ and $\left.\Delta \theta_{B}\right)$. By using 
the Jones vector notation $\left(\begin{array}{l}x \\ y\end{array}\right)$ the output field is given by

$$
E_{\text {out }}(\omega)=\left(\begin{array}{c}
1 \\
0
\end{array}\right) \cos \left(\frac{\Delta \theta_{A}-\Delta \theta_{B}}{2}\right) e^{i \frac{\Delta \theta_{A}+\Delta \theta_{B}}{2}} E_{\text {in }}(\omega) .
$$

Therefore, the intensity and phase of the transmitted light can be controlled independently by varying $\Delta \theta_{A}$ and $\Delta \theta_{B}$; a pulse pair is generated by setting these values so as to satisfy eq. (S2). We note that the actual retardation is doubled due to the reflection configuration of the setup.

The 2DES signal obtained by a pump-probe configuration is a sum of the two phase matching processes: ${ }^{9} k_{s}=-k_{1}+k_{2}+k_{3}$ and $k_{s}=+k_{1}-k_{2}+k_{3}$, where $k_{1}$ and $k_{2}$ stand for the wavevectors of two pump pulses (and then $k_{1}=k_{2}$ ) and $k_{3}$ is that of the probe pulse. Because the probe pulse also acts as a local oscillator (LO) in the 2DES with a pump-probe geometry, the phase of the signal $\phi_{\text {sig }}$ is given by ${ }^{8,10}$

$$
\phi_{\text {sig }}= \pm\left(\phi_{1}-\phi_{2}\right)+\phi_{\text {probe }}-\phi_{\mathrm{LO}}= \pm\left(\phi_{1}-\phi_{2}\right)+\phi_{\text {probe }}-\phi_{\text {probe }}= \pm\left(\phi_{1}-\phi_{2}\right),
$$

where $\phi_{\text {probe }}\left(\phi_{\mathrm{LO}}\right)$ is that of the probe $(\mathrm{LO})$ pulse.

The coherence time $\tau$ was scanned from 0 to $150 \mathrm{fs}$ with a scanning step $\Delta \tau$ of $3 \mathrm{fs}$ step. A rotating frame was used to make the scanning step meet the Nyquist frequency requirement: the phase difference between the pulse pair $\Delta \phi=\phi_{1}-\phi_{2}$ is modulated as a function of $\tau$ as $\Delta \phi=\omega_{r} \cdot \tau$, where $\omega_{r}$ is a reference frequency set to satisfy $\left(\omega_{0}-\omega_{r}\right) \cdot \Delta \tau<\pi / 2$. Here $\omega_{0}$ is a center frequency of the signal field.

The signal processing was done as follows: the pump induced change of the reflected probe spectrum,

$$
\Delta I\left(\phi_{1}, \phi_{2}\right)=\left(I\left(\phi_{1}, \phi_{2}\right)-I_{0}\right) / I_{0}
$$

was measured as a function of $\tau$ and $t$, where $I\left(\phi_{1}, \phi_{2}\right)$ is the probe spectrum with a pump pulse pair with phases of $\phi_{1}$ and $\phi_{2} ; I_{0}$ is the probe spectrum without pump irradiation. 
The 2DES signal $\Delta I_{2 D E S}$ was obtained by,

$$
\Delta I_{2 D E S}=\Delta I(0,0)-\Delta I(0, \pi)+\Delta I(\pi, \pi)-\Delta I(\pi, 0),
$$

which enables to eliminate unwanted scattering and transient absorption components. ${ }^{10}$

The incident angle of the excitation pulse was $\sim 67^{\circ}$. The pump-pair and probe pulses were focused onto the sample in UHV with $p$-polarization at slightly different incidence angles from each other. The fluences of the pump and probe pulses were $1 \mathrm{~mJ} / \mathrm{cm}^{-2}$ and $0.2 \mathrm{~mJ} / \mathrm{cm}^{-2}$, respectively. The reflected light of the probe was dispersed by a grating and focused on a multichannel array sensor (Hamamatsu S11639-01). The spectral data were acquired at $1 \mathrm{kHz}$ and processed in a computer. The time resolution of the measurement was estimated to be 30 fs from the autocorrelation of the pulse. 


\section{Characterizations of the tetracene film by a low-energy electron diffraction, ultraviolet photoemission, and photoluminescence spectroscopy}

Figure S3 shows typical LEED patterns of (a) the clean $\operatorname{Ir}(111)$, (b) a graphene (Gr) covered $\operatorname{Ir}(111)$, and (c) a tetracene film deposited on the graphene covered $\operatorname{Ir}(111)$. The graphene covered $\operatorname{Ir}(111)$ gives well-known satellite spots due to a moiré superstructure caused by a lattice mismatch between the graphene and $\operatorname{Ir}(111) \cdot{ }^{3}$ By the deposition of the multilayer tetracene on the graphene the moiré spots are replaced by some ring-like patterns with a weak six-fold azimuthal intensity modulation. By comparing with the LEED pattern of the $\operatorname{Ir}(111)$ and the known lattice constant of $\operatorname{Ir}(111),{ }^{11}$ the radii of the two smallest rings correspond to periodicities with lattice constants of $6.32 \AA$ and $8.08 \AA$. These are close to the reported lattice constants of tetracene crystal: $a=6.06 \AA$ and $b=7.94 \AA^{12}$ (see Figure S4 for the definition of the axes), indicating that the film consists of a layered structure in which molecules are arranged in a herringbone structure as in a bulk crystal with the molecular long-axis aligned normal to the surface.

(a)

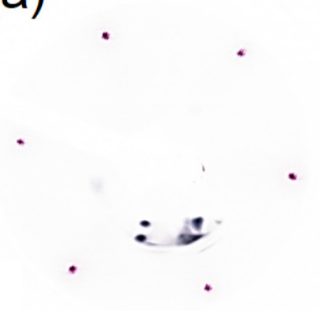

(b)

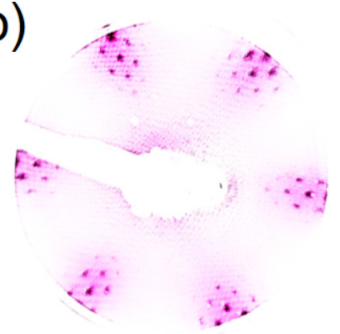

(c)

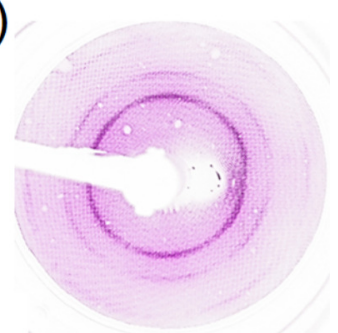

Figure S3: LEED patterns observed at $96 \mathrm{~K}$ for (a) $\operatorname{Ir}(111)$ clean surface (beam energy $98 \mathrm{eV})$; (b) $\mathrm{Gr} / \operatorname{Ir}(111)(111 \mathrm{eV})$; (c) 4nm thick tetracene/Gr/Ir(111) (55 eV).

Figure S5(a) shows He I ultraviolet photoemission spectra from the graphene (Gr) covered $\operatorname{Ir}(111)$ and the tetracene film deposited on the graphene substrate. The spectrum from the graphene covered $\operatorname{Ir}(111)$ exhibits a prominent peak at $1 \mathrm{eV}$ and some bumps at $\sim 4 \mathrm{eV}$, which are due to interband transitions in Ir. $^{13}$ The double peak structure apparent in the $4 \mathrm{eV}$ peak is due to the overlap of the $\pi$ band of the graphene. ${ }^{14}$ Upon 

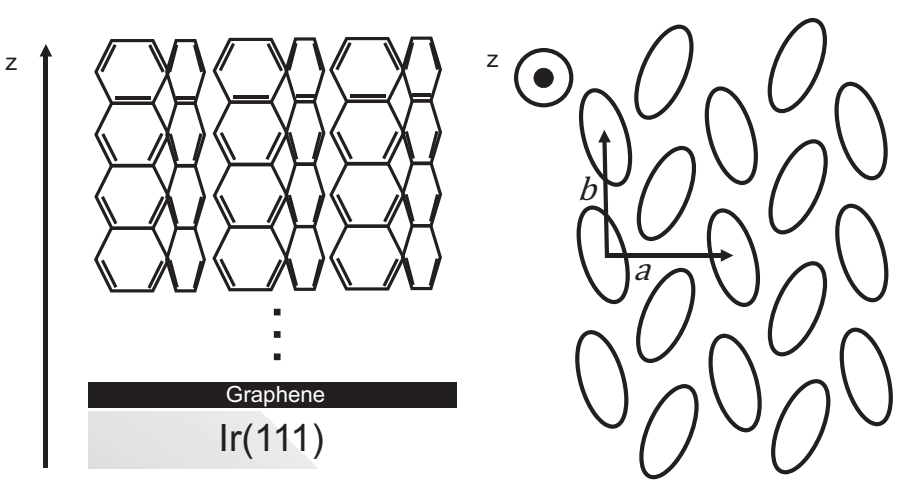

Figure S4: A schematic illustration of arrangement of tetracene molecules deduced from the LEED pattern. (Left) a side view indicating the molecules are stacked in layer-bylayer fashion. (Right) a top view in which an ellipsoid indicates a tetracene molecule. $z$ axis indicates the surface normal.

deposition of the tetracene thin film, those structures from the substrate are diminished and replaced by several peaks covering from $1 \mathrm{eV}$ to $7 \mathrm{eV}$. The spectral feature is consistent with UP spectra reported for 3 6 ML tetracene film deposited on a Si substrate. ${ }^{15}$ The binding energies of the series of peaks at $1.2 \mathrm{eV}, 2.4 \mathrm{eV}, 3.8 \mathrm{eV}$ and $5.2 \mathrm{eV}$ are in excellent agreement with the orbital binding energies of a tetracene film reported in the literature: the former three orbitals are $b_{1 g} \pi$ orbitals and the last one is ascribed to $a_{g} \sigma$ orbital. ${ }^{15}$

Figure S5(b) shows a photoluminescence spectrum of a $4 \mathrm{~nm}$ tetracene film deposited on $\mathrm{Gr} / \mathrm{Ir}(111)$ measured at $96 \mathrm{~K}$. The measurement was carried out with the same chamber by exciting the sample with a cw diode laser at $405 \mathrm{~nm}$. The luminescence from the sample was collected with the same optical setup depicted in Figure S1. The spectrum exhibits a prominent $0-0$ peak at $2.32 \mathrm{eV}$ and a weak $0-1$ band at $\sim 2.2 \mathrm{eV}$. These are consistent with the previous report on the emission spectrum of a tetracene film by Lim and co-workers. ${ }^{16}$ Notably, the full-width at half maximum of the $0-0$ band in this work $(\sim 41 \mathrm{meV})$ is significantly smaller than that in $\operatorname{ref}^{16}(\sim 78 \mathrm{meV}$, at $4 \mathrm{~K})$, indicating the reduced structural inhomogeneity of the ultrathin film on $\mathrm{Gr} / \operatorname{Ir}(111)$. 

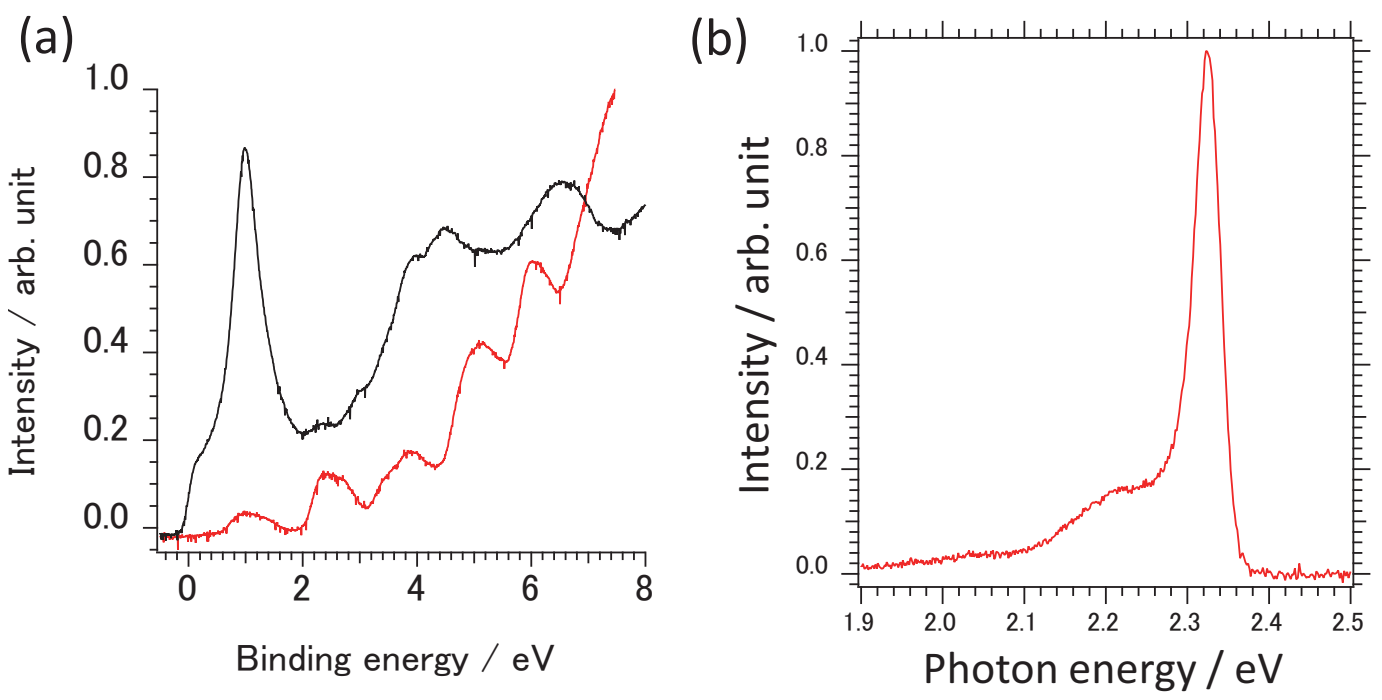

Figure S5: (a) UP spectra of $\mathrm{Gr} / \operatorname{Ir}(111)$ (black) and $4 \mathrm{~nm}$ thick tetracene/ $\mathrm{Gr} / \mathrm{Ir}(111)$ (red) at $96 \mathrm{~K}$. (b) A photoluminescence spectrum of a $4 \mathrm{~nm}$ thick tetracene film on $\mathrm{Gr} / \mathrm{Ir}(111)$. The excitation light source was a cw diode laser at $405 \mathrm{~nm}$. Sample temperature was $96 \mathrm{~K}$. 


\section{Numerical simulations of the optical reflectivity of a tetracene thin film}

The steady state and time-resolved reflectance spectra of the tetracene thin film are affected by optical interference with a multiple internal reflection due to the thin film structure. Here we describe the numerical analysis of the interference effect by using a transfer matrix method. ${ }^{4}$ Figure S6 shows a schematic illustration of the model used in the calculation. $N_{j}$ s are (complex) refractivity of materials in which $j=0$ corresponds to vacuum, $j=1$ denotes tetracene film, and $j=2$ denotes Ir substrate, respectively. $r_{j k}$ and $t_{j k}$ are Fresnel reflectivity and transmittance coefficients, respectively, at an interface between materials with $N_{j}$ and $N_{k}$. In this model the graphene layer is neglected because it gives a flat spectrum in the observation range and does not contribute to the reflectivity change induced by the tetracene deposition.

Reflectance and transmittance Fresnel coefficients for $p$-polarized light $r_{j k, p}, t_{j k, p}$ are defined as,

$$
r_{j k, p}=\frac{N_{k} \cos \theta_{j}-N_{j} \cos \theta_{k}}{N_{k} \cos \theta_{j}+N_{j} \cos \theta_{k}}, \quad t_{j k, p}=\frac{2 N_{j} \cos \theta_{j}}{N_{k} \cos \theta_{j}+N_{j} \cos \theta_{k}}
$$

A phase change $\beta$ experienced by light with vacuum wavelength of $\lambda_{0}$ transmitted through a tetracene film with a thickness of $d \mathrm{~nm}$ is given by

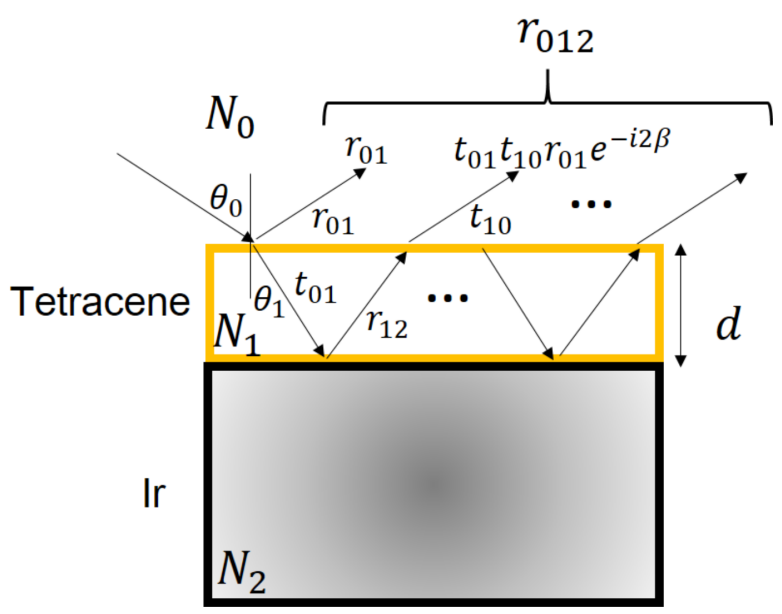

Figure S6: A schematic illustration of the model used in the transfer matrix calculation. 


$$
\beta=\frac{2 \pi d}{\lambda} N_{1} \cos \theta_{1}=\frac{2 \pi d}{\lambda} \sqrt{N_{1}^{2}-N_{0}^{2} \sin ^{2} \theta_{0}}
$$

The net reflection coefficient of the film $r_{012}$ is given by a summation of all the higher order multiple reflection process and given by

$$
\begin{aligned}
r_{012} & =r_{01}+t_{01} t_{10} r_{12} e^{-i 2 \beta}+t_{01} t_{10} r_{10} r_{12}^{2} e^{-i 4 \beta}+t_{01} t_{10} r_{10}^{2} r_{12}^{3} e^{-i 6 \beta}+\cdots \\
& =\frac{r_{01}+r_{12} e^{-i 2 \beta}}{1+r_{01} r_{12} e^{-i 2 \beta}}
\end{aligned}
$$

The $p$-polarization reflectivity of the tetracene film on the substrate $R_{012, p}$ is given by $R_{012, p}=\left|r_{012, p}\right|^{2}$. The reflectivity change induced by $x \mathrm{~nm}$ tetracene deposition is given by,

$$
\Delta R_{p}=\frac{R_{012, p}(d=x \mathrm{~nm})}{R_{012, p}(d=0 \mathrm{~nm})}
$$

$\Delta R_{p}$ corresponds to the reflectance change $I(\theta, E) / I(0, E)$ obtained from the experiment. Figure $\mathrm{S} 7$ shows simulated spectra, $\Delta R_{p}$, for various $d$ values by using the refractive indices of tetracene crystal reported in ref. ${ }^{17}$ and those of Ir given in ref. ${ }^{18}$ The calculations are consistent with the experiment (Figure 1(b) in the main text) that allows to estimate the nominal thickness of the film.

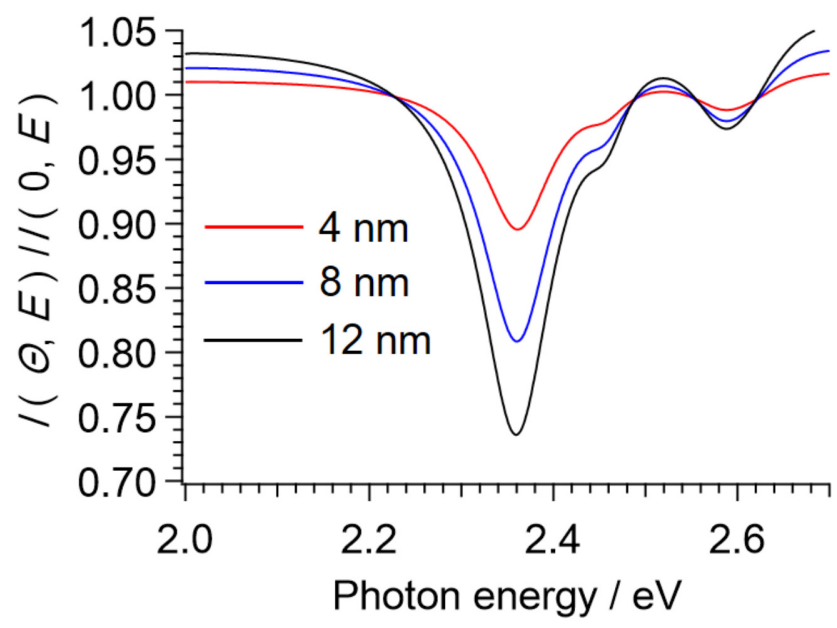

Figure S7: Simulated reflectivity change with tetracene deposition by using eq. (S11) for a film thickness $d=4 \mathrm{~nm}$ (red), $8 \mathrm{~nm}$ (blue), $12 \mathrm{~nm}$ (black). 


\section{Temperature dependence of LEED pattern}

Here we describe temperature dependence of the LEED pattern of the tetracene thin film. Figure S8(a)-(c) shows a series of LEED patterns of the tetracene film with various temperature. While the couple of ring patterns that reflects the in-plane herringbone structure of the molecule persist, their intensity drops with temperature increase as is plotted in Figure S8(d). We analyzed the temperature dependence of the diffraction intensity $I$ with the following equation, ${ }^{19,20}$

$$
I=\sum_{j, i} f_{j} \exp \left(-M_{j}\right) f_{i} \exp \left(-M_{i}\right) \exp \left(-2 \pi i \mathbf{K}\left(\mathbf{r}_{j}-\mathbf{r}_{i}\right)\right)+\sum_{j}\left(1-\exp \left(-2 M_{j}\right)\right) f_{j}^{2}
$$

where $M_{j}=2 \pi^{2}\left\langle\left(-\mathbf{K} \cdot \Delta \mathbf{r}_{j}\right)^{2}\right\rangle$ is Debye-Waller factor; $\Delta \mathbf{r}_{j}$ is a thermal lattice displacement; $\mathbf{r}_{j}$ is the equilibrium position of $j$ th atom; $f_{j}$ is the scattering factor of $j$ th atom; $\mathbf{K}$ is a scattering vector. With a harmonic approximation, the thermal lattice displacement is given by,

$$
\left\langle\Delta \mathbf{r}_{j}^{2}\right\rangle=\frac{\hbar}{2 m \omega} \operatorname{coth}\left(\frac{\hbar \omega}{2 k_{B} T}\right)
$$

where $m$ stands for the effective mass. Because the second term of eq. (S12) describes an inelastic scattering, we analyze the temperature dependence in Figure S8(d) with the first term of eq. (S12) with eq. (S13) by setting $\hbar \omega$ as a free parameter. The fitting result is plotted in Figure S8(d) with a blue curve and the optimum value of $\hbar \omega$ was found to be $235 \mathrm{~cm}^{-1}$. Because the deduced frequency is reasonable as a mean phonon frequency of tetracene solid, the temperature dependence of the LEED intensity can be understood as a conventional thermal effect. (Figure S8(e)). 

(a) $96 \mathrm{~K}$
(b) $167 \mathrm{~K}$
(c) $186 \mathrm{~K}$

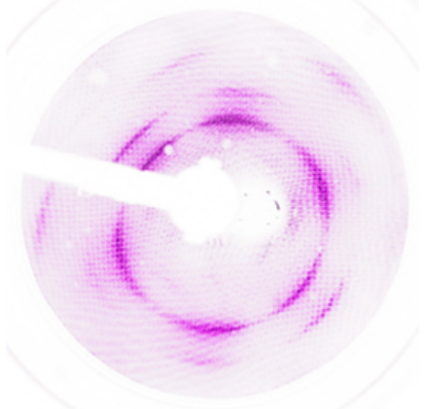

(d)

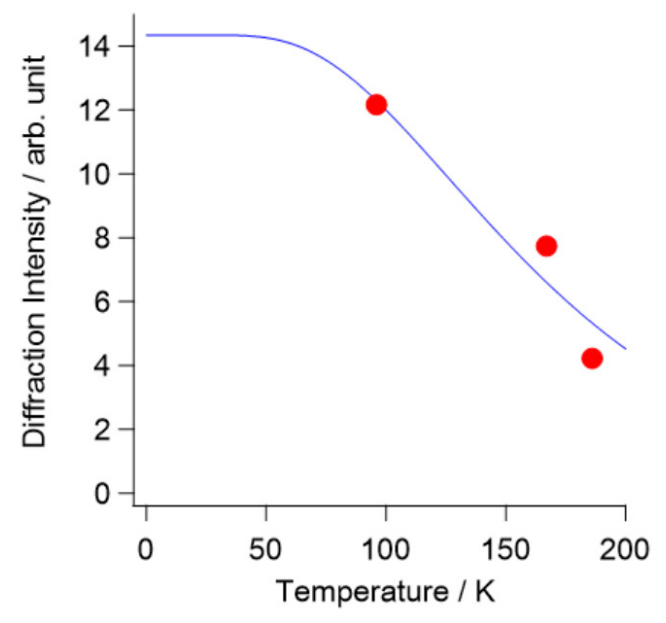

(e)

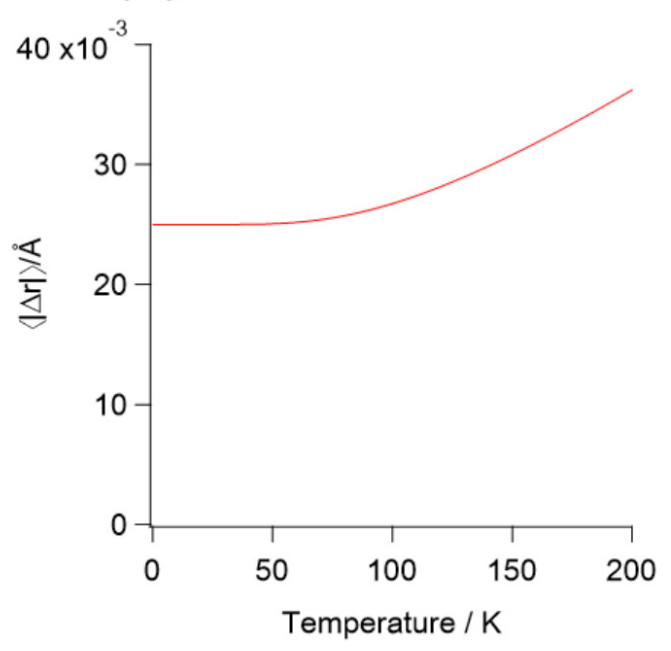

Figure S8: (a)-(c) Temperature dependence of the LEED patterns from $4 \mathrm{~nm}$ tetracene film. (a) $96 \mathrm{~K}$, (b) $167 \mathrm{~K}$, and (c) $186 \mathrm{~K}$. (d) (Red circles) temperature dependence of the intensity of the ring structure of the LEED pattern. (Solid curve) a fitting results with eq. (S13). (e) Root mean square of the thermal lattice displacement estimated with eq. (S13). 


\section{Estimation of $N_{c o h}$ by a two-dimensional Frenkel ex- citon model}

Here we extract $N_{c o h}$ of the tetracene film from the $2 \mathrm{D}$ signals based on a twodimensional Frenkel exciton model with $L \times L$ sites $\left(N_{c o h}=L^{2}\right) .{ }^{21}$ The eigenfunction of the exciton is given by,

$$
\left|k_{1}, k_{2}\right\rangle \propto \sum_{n, m} \sin \frac{\pi k_{1} n}{L+1} \sin \frac{\pi k_{2} m}{L+1}|n, m\rangle
$$

where $k_{1}, k_{2}$ denote wave vectors of exciton; $|n, m\rangle$ stand for a local excitation state in which the molecule at the site $(n, m)$ is excited while the others are in the ground state. With the nearest neighbor excitonic coupling of $J$, the eigenenergy of the model is given by,

$$
E_{k_{1}, k_{2}}=\omega_{0}-2 J\left(\cos \frac{\pi k_{1}}{L+1}+\cos \frac{\pi k_{2}}{L+1}\right)
$$

By expanding $E_{k_{1}, k_{2}}$ around the bottom of the band with respect to $k_{i}$, we obtain

$$
E_{k_{1}, k_{2}}=\omega_{0}-4 J+\frac{\pi^{2} J}{(L+1)^{2}}\left(k_{1}^{2}+k_{2}^{2}\right)
$$

The energy difference between GSB and ESA $\Delta E$ is given by,

$$
\Delta E=E_{2,1}-E_{1,1}=E_{1,2}-E_{1,1}=\frac{3 \pi^{2} J}{(L+1)^{2}}
$$

From the 2DES plots, the average value of $\Delta E$ in the range in which the GSB amplitude is larger than 0.75 is estimated for each population time. By assuming that $J=280 \mathrm{~cm}^{-1}$ in line with the previous work on the spectral analysis of a tetracene film, ${ }^{16}$ temperature dependence of $N_{c o h}$ as a function of the population time $t$ is estimated (Figure 3(b) in the main text). 


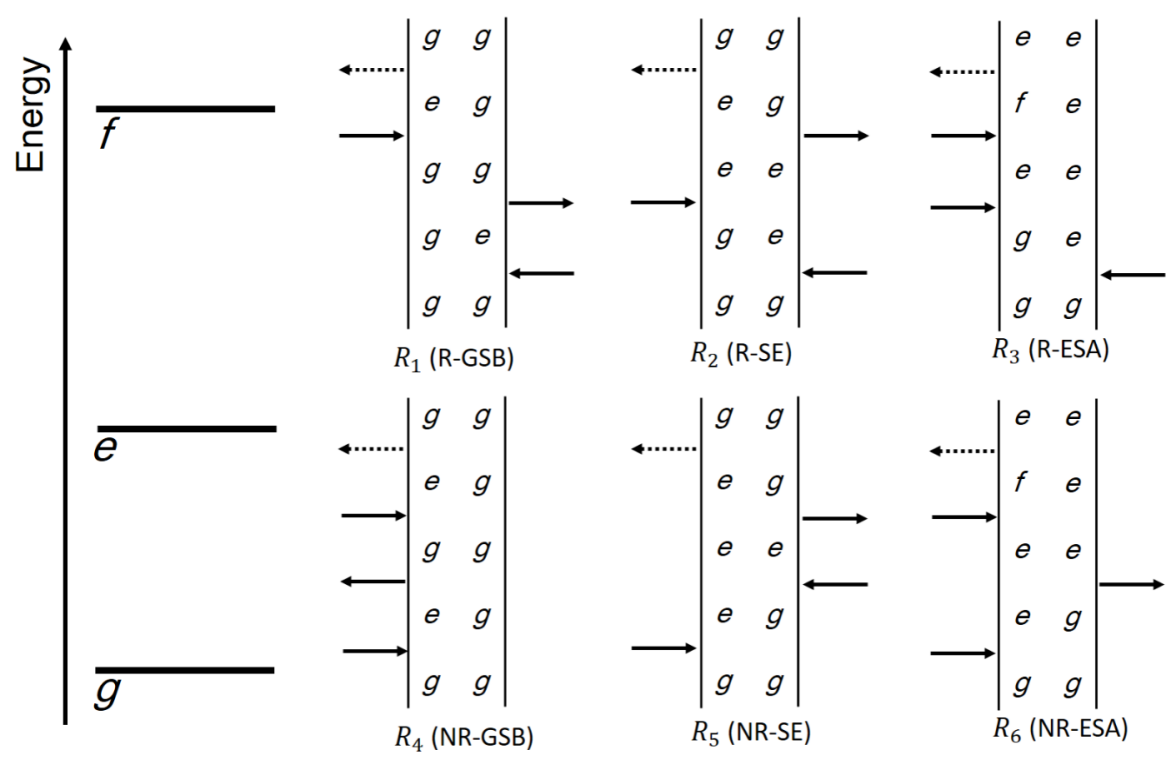

Figure S9: (Left) Schematic illustration of the three level system assumed in the simulation. (Right) Feynman diagrams of the response functions given in eq. (S20)-(S23).

\section{Numerical simulation of the 2DES spectra used for deducing FFCF parameters}

In this section, we describe the simulation procedure used to extract the parameters of FFCF in eq. (2) in the main text from the 2DES data. In this analysis. we assume the following multi-component form as the line shape function of the tetracene thin film,

$$
g(t)=\sum_{j=1}^{2} \frac{\Delta_{j}^{2}}{\Lambda_{j}^{2}}\left(e^{-\Lambda_{j} t}+\Lambda_{j} t-1\right)+\frac{1}{2} \Delta_{s d}^{2} t^{2}
$$

where $\Delta_{j}$ and $\Lambda_{j}^{-1}$ are the amplitude and the correlation time of the $j$ th fluctuation component in the excitation energy $\omega_{e g}$, respectively; $\Delta_{s d}$ is the static disorder. Once the parameters $\Delta_{j}, \Lambda_{j}^{-1}$, and $\Delta_{s d}$ are given, a purely absorptive 2DES signal can be simulated by assuming a three level electronic system as follows: ${ }^{10,22}$

$$
S_{2 D E S}\left(\omega_{1}, \omega_{3}, t_{2}\right)=\operatorname{Re}\left(\sum_{i=1 \sim 6} \int_{0}^{\infty} e^{i s \omega_{1} t_{1}} \int_{0}^{\infty} e^{i \omega_{3} t_{3}} R_{i}^{(3)}\left(t_{3}, t_{2}, t_{1}\right)\right)
$$


where $s=-1$ for $i=1 \sim 3$ and $s=+1$ for $i=4 \sim 6$; the third order response function $R_{i}^{(3)}$ is given by, ${ }^{22,23}$

$$
\begin{aligned}
& R_{1}^{(3)}(t)=R_{2}^{(3)}(t)=\mu_{e g}^{4} e^{i \omega_{e g}\left(t_{1}-t_{3}\right)} e^{-g\left(t_{1}\right)+g\left(t_{2}\right)-g\left(t_{3}\right)-g\left(t_{1}+t_{2}\right)-g\left(t_{2}+t_{3}\right)+g\left(t_{1}+t_{2}+t_{3}\right)}, \\
& R_{3}^{(3)}(t)=\mu_{e g}^{2} \mu_{f e}^{2} e^{i\left(\omega_{e g} t_{1}-\omega_{f e} t_{3}\right)} e^{-g\left(t_{1}\right)+g\left(t_{2}\right)-g\left(t_{3}\right)-g\left(t_{1}+t_{2}\right)-g\left(t_{2}+t_{3}\right)+g\left(t_{1}+t_{2}+t_{3}\right)}, \\
& R_{4}^{(3)}(t)=R_{5}^{(3)}(t)=\mu_{e g}^{4} e^{i \omega_{e g}\left(t_{1}+t_{3}\right)} e^{-g\left(t_{1}\right)-g\left(t_{2}\right)-g\left(t_{3}\right)+g\left(t_{1}+t_{2}\right)+g\left(t_{2}+t_{3}\right)-g\left(t_{1}+t_{2}+t_{3}\right)}, \\
& R_{6}^{(3)}(t)=\mu_{e g}^{2} \mu_{f e}^{2} e^{i\left(\omega_{e g} t_{1}+\omega_{f e} t_{3}\right)} e^{-g\left(t_{1}\right)-g\left(t_{2}\right)-g\left(t_{3}\right)+g\left(t_{1}+t_{2}\right)+g\left(t_{2}+t_{3}\right)-g\left(t_{1}+t_{2}+t_{3}\right)}
\end{aligned}
$$

where $\mu_{e g}\left(\mu_{f e}\right)$ is the transition dipole between $g$ and $e(e$ and $f)$. The Feynman diagram of each component is depicted in Figure S9.

We carried out a numerical survey for the parameter sets of $\Lambda_{i}^{-1}$, and $\Delta_{s d}$ which give a similar nodal line slope dynamics as is observed experimentally. However, $\Delta_{i}$ cannot be optimized with this procedure because NLS does not give an absolute value of the FFCF. $\Delta_{i}$ can be determined by using the fact that the linear absorption spectrum $S_{l}(\omega)$ is given by,

$$
S_{l}(\omega) \propto \operatorname{Re} \int_{0}^{\infty} e^{i\left(\omega-\omega_{e g}\right) t} e^{-g(t)}
$$

$\Delta_{i}$ determines the magnitude of $g(t)$, hence affects the linewidth of $S_{l}(\omega)$. The optimum value for $\Delta_{i}$ is found by comparing $S_{l}(\omega)$ calculated by using eq. (S24) with the experimental absorption spectrum of the film. Figure S10 shows the fitting results and Figure S11 shows the simulated 2DES signals by using thus deduced parameters tabulated in Table 1 in the main text. We note that the sign of $S_{2 D E S}$ is opposite to that of $\Delta I_{2 D E S}$ plotted in Figure 2 in the main text because of the sign convention in eq. (S6). 


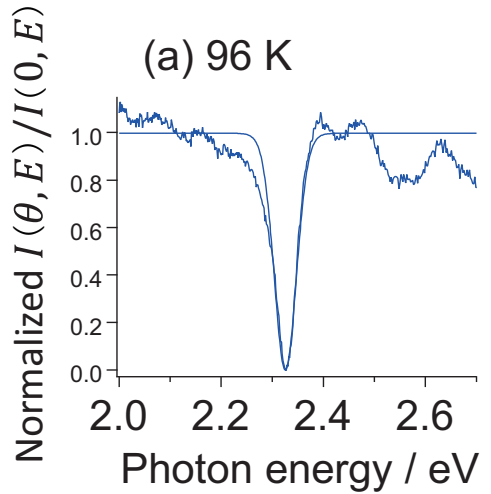

(b) $167 \mathrm{~K}$
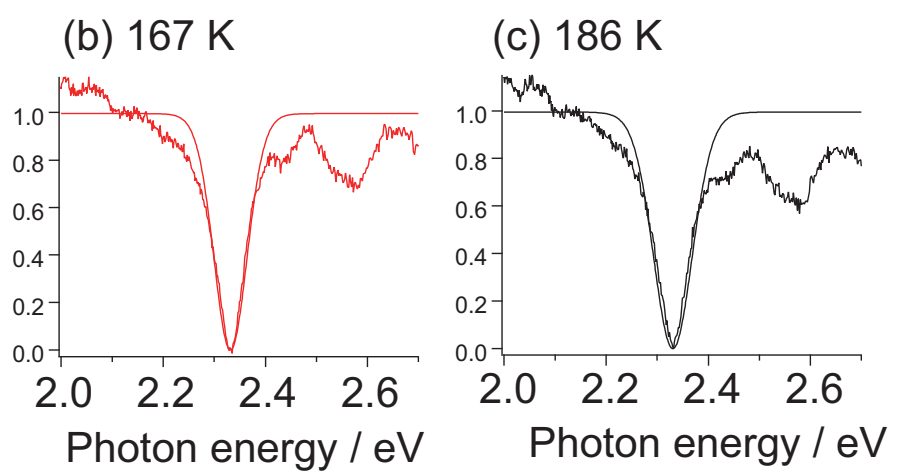

Figure S10: Temperature dependence of the steady state reflectivity change induced by the tetracene film deposition. (a) $96 \mathrm{~K}$, (b) $167 \mathrm{~K}$, and (c) $186 \mathrm{~K}$. The negative peaks at $2.32 \mathrm{eV}$ corresponds to the lowest exciton absorption. Thin solid curves show simulation spectra of the linear absorption $S(\omega)$ by using eq. (S24). We note that the simulation spectra were sign inverted and scaled to the experiments.

(a) $96 \mathrm{~K}, 50 \mathrm{fs}$

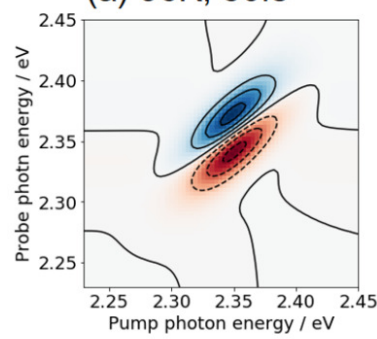

(d) $186 \mathrm{~K}, 50 \mathrm{fs}$

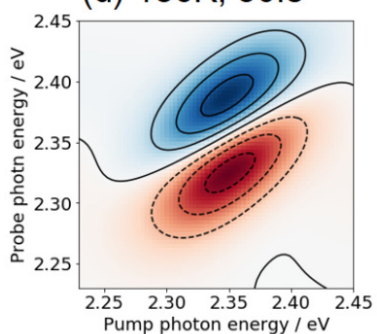

(b) $96 \mathrm{~K}, 200 \mathrm{fs}$

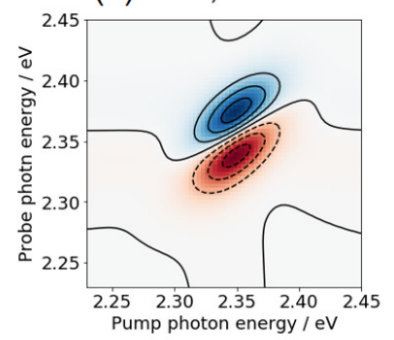

(e) $186 \mathrm{~K}, 200 \mathrm{fs}$

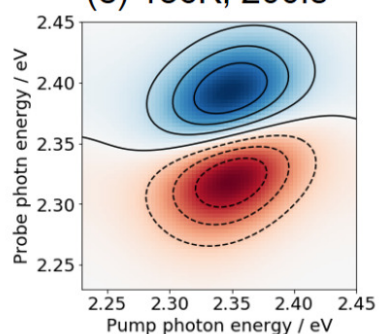

(c) $96 \mathrm{~K}, 1000 \mathrm{fs}$

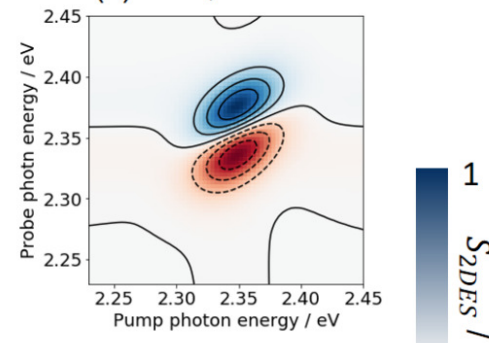

(f) $186 \mathrm{~K}, 1000 \mathrm{fs}$

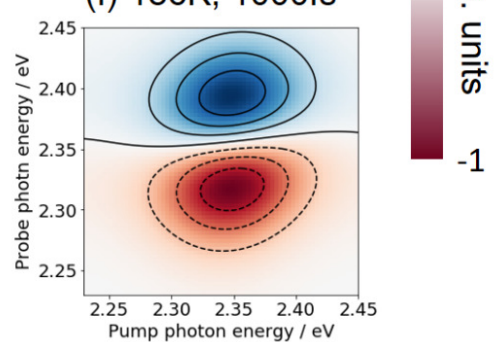

Figure S11: 2DES signals simulated by using eq. (S19) with parameters for $g(t)$ listed in Table 1 in the main text. In the simulation, we assumed that $\mu_{e g}=\mu_{f e}=1, \omega_{e g}=2.35$ $\mathrm{eV}$, and $\omega_{f e}=2.37 \mathrm{eV}$. The temperature and the population time are indicated in the figure. 


\section{Anharmonic coupling model of the correlation func- tion}

Here we describe a possible displaced oscillator model that can explain the observed temperature dependence of the amplitude of the $\Lambda_{2}^{-1}=80 \mathrm{fs}$ component in the correlation function. The model employs two normal modes: one is a high frequency intramolecular mode $Q_{a}$ with a displacement in its equilibrium position upon electronic excitation (mode $a)$; the other is a low frequency phonon mode $Q_{b}$ with a negligible displacement (mode $b$ ) (see Figure $4(\mathrm{c})$ in the main text). $Q_{a}$ corresponds to a carbon-carbon stretching mode of tetracene at $\sim 1400 \mathrm{~cm}^{-1}$, which is known for the prominent electron-vibrational coupling upon the $S_{0} \rightarrow S_{1}$ transition. We propose that the $\Lambda_{2}$ component is an energy gap fluctuation along with the $Q_{a}$ coordinate and the 80 fs correlation time is due to the damping of this mode $a$.

We demonstrate that the peculiar temperature dependence of $\Delta_{2}^{2}$ (the amplitude of the $\Lambda_{2}$ component) is accounted for by considering an anharmonic coupling between $Q_{a}$ and $Q_{b}$ by which the thermal occupation of the latter mode determines $\Delta_{2}^{2}$. The two modes are treated as harmonic oscillators with an intermode coupling with a quartic order. The Hamiltonian of the system is given by,

$$
\begin{aligned}
H_{g} & =\hbar \omega_{a} a_{a}^{\dagger} a_{a}+\hbar \omega_{b} a_{b}^{\dagger} a_{b}+W\left(a_{a}^{\dagger}+a_{a}\right)^{2}\left(a_{b}^{\dagger}+a_{b}\right)^{2} \\
H_{e} & =\hbar \omega_{e g}^{0}+\hbar \omega_{1} a_{a}^{\dagger} a_{a}+\hbar \omega_{1}\left(a_{a}^{\dagger}+a_{a}\right) D+\frac{1}{2} \hbar \omega_{1} D^{2} \\
& +\hbar \omega_{2} a_{b}^{\dagger} a_{b}+W\left(a_{a}^{\dagger}+a_{a}+D\right)^{2}\left(a_{b}^{\dagger}+a_{b}\right)^{2},
\end{aligned}
$$

where $H_{g}\left(H_{e}\right)$ is the Hamiltonian of the electronic ground (excited) state; $\hbar \omega_{i}$ is the vibrational quanta of the mode $i(=a, b) ; a_{i}^{\dagger}\left(a_{i}\right)$ is creation (annihilation) operator of the mode $i$; $D$ is the displacement of the mode a (see Figure 4(c) in the main text); $W$ is the coefficient of the anharmonic term. We note that the damping of each modes are not incorporated in the Hamiltonian for simplicity. 
We define an energy gap operator $U$ as,

$$
\begin{aligned}
U & =H_{e}-H_{g}-\hbar \omega_{e g}^{0}-\frac{1}{2} \hbar \omega_{1} D^{2} \\
& =\hbar \omega_{a} D\left(a_{a}^{\dagger}+a_{a}\right)+W D^{2}\left(a_{b}^{\dagger}+a_{b}\right)^{2}+2 W D\left(a_{a}^{\dagger}+a_{a}\right)\left(a_{b}^{\dagger}+a_{b}\right)^{2},
\end{aligned}
$$

which leads to a time-correlation function $C_{f}(t),{ }^{22}$

$$
\begin{aligned}
C_{f}(t) & =\left\langle\exp \left(i H_{g} t / \hbar\right) U \exp \left(-i H_{g} t / \hbar\right) U\right\rangle \\
& =\operatorname{Tr}\left[\exp \left(i H_{g} t / \hbar\right) U \exp \left(-i H_{g} t / \hbar\right) U \rho_{g}\right]
\end{aligned}
$$

where $\rho_{g}=e^{-H_{g} / k_{B} T} /\left\langle e^{-H_{g} / k_{B} T}\right\rangle$, with the Boltzmann constant $k_{B}$.

On evaluating eq. (S28), we employ an approximation of

$$
\exp \left( \pm i H_{g} t / \hbar\right) \simeq \exp \left( \pm i \omega_{1} t a_{a}^{\dagger} a_{a} \pm i \omega_{2} t a_{b}^{\dagger} a_{b}\right)
$$

then, by using the following identity: ${ }^{24}$

$$
\begin{aligned}
\mathrm{e}^{i \omega_{i} t a_{i}^{\dagger} a_{i}} a_{i}^{m} \mathrm{e}^{-i \omega_{i} t a_{i}^{\dagger} a_{i}} & =a_{i}^{m} \mathrm{e}^{-i m \omega_{i} t}, \\
\mathrm{e}^{i \omega_{i} t a_{i}^{\dagger} a_{i}} a_{i}^{\dagger m} \mathrm{e}^{-i \omega_{i} t a_{i}^{\dagger} a_{i}} & =a_{i}^{\dagger m} \mathrm{e}^{i m \omega_{i} t}, \\
\mathrm{e}^{i \omega_{i} t a_{i}^{\dagger} a_{i}} a_{i}^{\dagger} a_{i} \mathrm{e}^{-i \omega_{i} t a_{i}^{\dagger} a_{i}} & =a_{i}^{\dagger} a_{i},
\end{aligned}
$$

where $m$ is a positive integer, surviving terms in eq. (S28) are,

$$
\begin{aligned}
C_{f}(t) & =2\left(\hbar \omega_{a}\right)^{2} D^{2} \mathrm{e}^{ \pm i \omega_{a} t}\left\langle a_{a}^{\dagger} a_{a}\right\rangle \\
& +2 \hbar \omega_{a} W D^{2} \mathrm{e}^{ \pm i \omega_{a} t}\left\langle a_{a}^{\dagger} a_{a} a_{b}^{\dagger} a_{b}\right\rangle \\
& +\sum_{k=0, \pm 2} 4 W^{2} D^{2} \mathrm{e}^{ \pm i \omega_{a} t+i k \omega_{b} t}\left\langle a_{a}^{\dagger} a_{a} a_{b}^{\dagger} a_{b} a_{b}^{\dagger} a_{b}\right\rangle .
\end{aligned}
$$

We note that the thermal average of the last term results in,

$$
\left\langle a_{a}^{\dagger} a_{a} a_{b}^{\dagger} a_{b} a_{b}^{\dagger} a_{b}\right\rangle=\left\langle n\left(\omega_{a}\right)\right\rangle\left\langle n\left(\omega_{b}\right)^{2}\right\rangle
$$


where $n(\omega)$ is an occupation of the mode $\hbar \omega$.

Therefore, this model demonstrates that the quartic coupling induces fluctuations in the gap energy with $\sim \omega_{a}$ frequency (note that $\omega_{b} \ll \omega_{a}$ is assumed) that shows a temperature dependence proportional to $\left\langle n\left(\omega_{a}\right)\right\rangle\left\langle n\left(\omega_{b}\right)^{2}\right\rangle$. Since $\left\langle n\left(\omega_{a}\right)\right\rangle$ is almost constant in the experimental temperature range, the observed amplitude follows a temperature dependence of $\left\langle n\left(\omega_{b}\right)^{2}\right\rangle$ (Figure $4(\mathrm{~b})$ in the main text).

This effect stems from that the curvature of the $Q_{b}$ potential differs between the ground and the excited state at around the Frank-Condon region due to the quartic coupling. This effectively enhances the amplitude of the energy-gap fluctuations along $Q_{a}$ with the thermal population of $Q_{b}$. We note that the thermal population of the high frequency mode $Q_{a}$ is negligible at $96-186 \mathrm{~K}$ and the fluctuations by the mode is due to its zero-point motion. The anharmonic coupling with the low frequency mode $Q_{b}$ helps to enhance the contribution of the high-frequency mode, leading to the steep temperature dependence of $\Delta_{2}^{2}$.

We further note that the above analysis shows that the amplitude of the fluctuations is proportional to $W^{2} D^{2}$. Therefore, a normal mode with a prominent exciton-phonon coupling with large $D$, that is the intramolecular $\mathrm{C}-\mathrm{C}$ stretching mode, is the most probable primary mode for the energy gap fluctuation. In addition, this leads that the amplitude also scales with $1 / \sqrt{N_{c o h}}$ because $D^{2} \propto \lambda \propto 1 / \sqrt{N_{c o h}}$. Indeed, incorporating the temperature dependence of $1 / \sqrt{N_{c o h}}$ found in Figure 3(a) in the main text gives a sufficient agreement between the simulation and the experiment as is shown in Figure 4(b) in the main text.

Furthermore, the currently proposed mechanism could be operational in other molecular systems as well. Fidder et al. have carried out a photon echo study on pseudoisocyanine $J$ aggregate and successfully extracted the exciton energy-gap correlation function: they found that the correlation time ( $\tau_{c}$ in their notation) varies from 150 fs to 60 fs from $25 \mathrm{~K}$ to $77 \mathrm{~K}$, while the fluctuation amplitude $\Delta$ increases about three times with temperature in the same temperature range. ${ }^{25}$ The time scale of $\tau_{c}$ and the almost linear temperature dependence of $\Delta$ (thus quadratic dependence of $\Delta^{2}$ ) found in their study 
is qualitatively similar to those of the $\Lambda_{2}$ component in the current work. Although a conventional linear coupling with a low-frequency phonon bath has been considered as the underlying mechanism in ref., ${ }^{25}$ we rather note that the anharmonic coupling model proposed in this work can be valid in the $J$ aggregate. Interestingly, a recent 2DES study of a cyanine dye monomer in solution has shown a spectral evolution within 100 fs and the dynamics has been ascribed to fluctuations with a $1300 \mathrm{~cm}^{-1}$ intramolecular mode. ${ }^{26}$ We consider that this is the case not only for the monomer in the solution but also for the $J$ aggregate, and then, the observed temperature dependence of $\Delta$ in ref. ${ }^{25}$ is only possible with the anharmonic coupling model proposed in the current work. Consequently, the mechanism proposed in this study would work in other molecular systems which possesses an intramolecular mode with a large exciton-vibration coupling.

\section{References}

(1) Watanabe, K.; Matsumoto, Y.; Yasuike, T.; Nobusada, K. Adsorbate-Localized versus Substrate-Mediated Excitation Mechanisms for Generation of Coherent Cs-Cu Stretching Vibration at $\mathrm{Cu}(111)$. J. Phys. Chem. A 2011, 115, 9528-9535.

(2) Ino, D.; Watanabe, K.; Takagi, N.; Matsumoto, Y. Electron Transfer Dynamics from Organic Adsorbate to a Semiconductor Surface: Zinc Phthalocyanine on $\mathrm{TiO}_{2}(110)$. J. Phys. Chem. B 2005, 109, 18018-18024.

(3) Petrovič, M.; Rakic, I. S.; Runte, S.; Busse, C.; Sadowski, J. T.; Lazic, P.; Pletikosic, I.; Pan, Z. H.; Milun, M.; Pervan, P. et al. The Mechanism of Cesium Intercalation of Graphene. Nat. Commun. 2013, 4, 2772.

(4) Takahashi, S.; Watanabe, K.; Matsumoto, Y. Singlet Fission of Amorphous Rubrene Modulated by Polariton Formation. J. Chem. Phys. 2019, 151.

(5) Miyata, K.; Kurashige, Y.; Watanabe, K.; Sugimoto, T.; Takahashi, S.; Tanaka, S.; Takeya, J.; Yanai, T.; Matsumoto, Y. Coherent Singlet Fission Activated by Symmetry Breaking. Nat. Chem. 2017, 9, 983-989. 
(6) Weiner, A. Femtosecond Pulse Shaping Using Spatial Light Modulators. Rev. Sci. Instrum. 2000, 71, 1929-1960.

(7) Takahashi, S.; Watanabe, K. Decoupling from a Thermal Bath via Molecular Polariton Formation. J. Phys. Chem. Lett. 2020, 11, 1349-1356.

(8) Shim, S.-H.; Zanni, M. T. How to Turn Your Pump-Probe Instrument into a Multidimensional Spectrometer: 2D IR and Vis Spectroscopies via Pulse Shaping. Phys. Chem. Chem. Phys. 2009, 11, 748-761.

(9) DeFlores, L. P.; Nicodemus, R. A.; Tokmakoff, A. Two-dimensional Fourier Transform Spectroscopy in the Pump-probe Geometry. Opt. Lett. 2007, 32, 2966-2968.

(10) Hamm, P.; Zanni, M. Concepts and Methods of 2D Infrared Spectroscopy; Cambridge Univ. Press: Cambridge, UK, 2011.

(11) N'Diaye, A. T.; Coraux, J.; Plasa, T. N.; Busse, C.; Michely, T. Structure of Epitaxial Graphene on $\operatorname{Ir}(111)$. New J. Phys. 2008, 10, 043033.

(12) Oehzelt, M.; Aichholzer, A.; Resel, R.; Heimel, G.; Venuti, E.; Della Valle, R. Crystal Structure of Oligoacenes Under High Pressure. Phys. Rev. B 2006, 74, 104103.

(13) Van der Veen, J.; Himpsel, F.; Eastman, D. Experimental Energy Dispersions for Valence and Conduction Bands of Iridium. Phys. Rev. B 1980, 22, 4226.

(14) Varykhalov, A.; Marchenko, D.; Scholz, M.; Rienks, E.; Kim, T.; Bihlmayer, G.; Sánchez-Barriga, J.; Rader, O. Ir (111) Surface State with Giant Rashba Splitting Persists under Graphene in Air. Phys. Rev. Lett. 2012, 108, 066804.

(15) Mao, H.; Guan, D.; Chen, M.; Dou, W.; Song, F.; Zhang, H.; Li, H.; He, P.; Bao, S. The Chemisorption of Tetracene on Si (100)-2×1 Surface. J. Chem. Phys. 2009, 131, 044703.

(16) Lim, S.-H.; Bjorklund, T. G.; Spano, F. C.; Bardeen, C. J. Exciton Delocalization and Superradiance in Tetracene Thin Films and Nanoaggregates. Phys. Rev. Lett. 2004, 92, 107402. 
(17) Tavazzi, S.; Raimondo, L.; Silvestri, L.; Spearman, P.; Camposeo, A.; Polo, M.; Pisignano, D. Dielectric Tensor of Tetracene Single Crystals: The Effect of Anisotropy on Polarized Absorption and Emission Spectra. J. Chem. Phys. 2008, $128,154709$.

(18) Palik, E. D. Handbook of Optical Constants of Solids; Academic press, 1985.

(19) Allen, R. E.; De Wette, F. W. Mean-Square Amplitudes of Vibration at a Surface. Phys. Rev. 1969, 188, 1320-1323.

(20) McKinney, J. T.; Jones, E. R.; Webb, M. B. Surface Lattice Dynamics of Silver. II. Low-Energy Electron Thermal Diffuse Scattering. Phys. Rev. 1967, 160, 523-530.

(21) Arias, D. H.; Stone, K. W.; Vlaming, S. M.; Walker, B. J.; Bawendi, M. G.; Silbey, R. J.; Bulovic, V.; Nelson, K. A. Thermally-Limited Exciton Delocalization in Superradiant Molecular Aggregates. J. Phys. Chem. B 2013, 117, 4553-4559.

(22) Mukamel, S. Principles of Nonlinear Optical Spectroscopy; Oxford Univ. Press: New York, 1995.

(23) Valkunas, L.; Abramavicius, D.; Mančal, T. Molecular Excitation Dynamics and Relaxation; Wiely-VCH, 2013.

(24) Louisell, W. H. Quantum Statistical Properties of Radiation; John Wiley \& Sons Inc, 1973.

(25) Fidder, H.; Knoester, J.; Wiersma, D. A. Optical Properties of Disordered Molecular Aggregates: a Numerical Study. J. Chem. Phys. 1991, 95, 7880-7890.

(26) Nemeth, A.; Lukeš, V.; Sperling, J.; Milota, F.; Kauffmann, H. F.; Mančal, T. Twodimensional Electronic Spectra of an Aggregating Dye: Simultaneous Measurement of Monomeric and Dimeric Line-shapes. Phys. Chem. Chem. Phys. 2009, 11, 59865997. 